\title{
Repeat Stent Placement through Lateral Fenestration of the Existing Dysfunctioning DIPS Stent Graft: An Alternative to Parallel TIPS/DIPS Procedure in a Case of Blocked Primary TIPS/DIPS
}

\author{
Karan Manoj Anandpara ${ }^{1}$ Amar Mukund ${ }^{1}$ Ravindran Ramalingam ${ }^{1}$ Rakhi Maiwall ${ }^{2}$ \\ ${ }^{1}$ Department of Interventional Radiology, Institute of Liver and \\ Biliary Sciences, New Delhi, India \\ 2Department of Hepatology, Institute of Liver and Biliary Sciences, \\ Address for correspondence Amar Mukund, MD, Department of \\ Interventional Radiology, Institute of Liver and Biliary Sciences, D-1, \\ Vasant Kunj, New Delhi, India (e-mail: dramarmukund@gmail.com).
} New Delhi, India

J Clin Interv Radiol ISVIR:2020;4:118-121

\begin{abstract}
Keywords

- transjugular intrahepatic portosystemic shunt (TIPS)

- direct intrahepatic portosystemic shunt (DIPS)

- TIPS revision

- portal hypertension

- blocked TIPS

A complication of transjugular and direct intrahepatic portosystemic stent (TIPS and DIPS) graft is stent blockage. Routinely described procedures for shunt revision include angioplasty, deployment of endoprosthesis, catheter-directed thrombolysis, or rarely performing a second parallel TIPS/DIPS. We describe a case of hepatic vein outflow tract obstruction who presented with DIPS blockage. We performed a revision where a new stent was placed by a lateral puncture through the fenestration of the existing dysfunctioning DIPS stent graft. In our opinion, this alternate technique has theoretical advantages over the conventionally described parallel TIPS/DIPS as it prevents the creation of a completely new long hepatic parenchymal tract.
\end{abstract}

\section{Introduction}

Transjugular and direct intrahepatic portosystemic shunt (TIPS/DIPS) is a routinely described procedure for the treatment of complications of portal hypertension. Over the past decade, covered stents have replaced uncovered bare metallic stents during TIPS/DIPS. Nonetheless, stent blockage and dysfunction are known complications. Various treatment options are available, including rarely performing a second TIPS procedure with a new puncture track parallel to the original stent called parallel TIPS/DIPS. We describe an alternate technique for TIPS/DIPS revision where a new stent is placed through lateral fenestration of the existing dysfunctioning blocked DIPS stent graft. We believe this can have theoretical advantages over the parallel TIPS/DIPS technique.

\section{Case Report}

A 36-year-old male patient presented with gradually increasing abdominal distension of 3-week duration. The patient had his index presentation 6 years earlier when he was diagnosed with hepatic venous outflow tract obstruction and associated chronic liver disease. He underwent DIPS stent-graft placement in 2013 and was lost to follow-up. Now the patient again presented with gradually increasing ascites. On ultrasound imaging, DIPS stent was seen in situ; however, it showed absent flow on Doppler suggestive of DIPS blockage. A DIPS revision/recanalization was therefore planned.

The left internal jugular vein was accessed (since the right internal jugular vein was chronically thrombosed) using standard angiographic techniques and a $10 \mathrm{~F}$ vascular access sheath (Cook Medical) was placed ( - Fig. 1A). Right atrial pressure was $6 \mathrm{~mm} \mathrm{Hg}$ and inferior vena cava (IVC) pressure was $8 \mathrm{~mm} \mathrm{Hg}$. Failed attempts were used to access the DIPS stent using a multipurpose catheter (Boston Scientific) and hydrophilic guidewire (Teremo); long sheath, stiffening cannula, and the needle of the Rösch-Uchida Transjugular Liver Access Set (RUPS; Cook Medical). However, it was not possible to access the stent graft probably due to long-standing chronic thrombosis and also probably due to difficult 

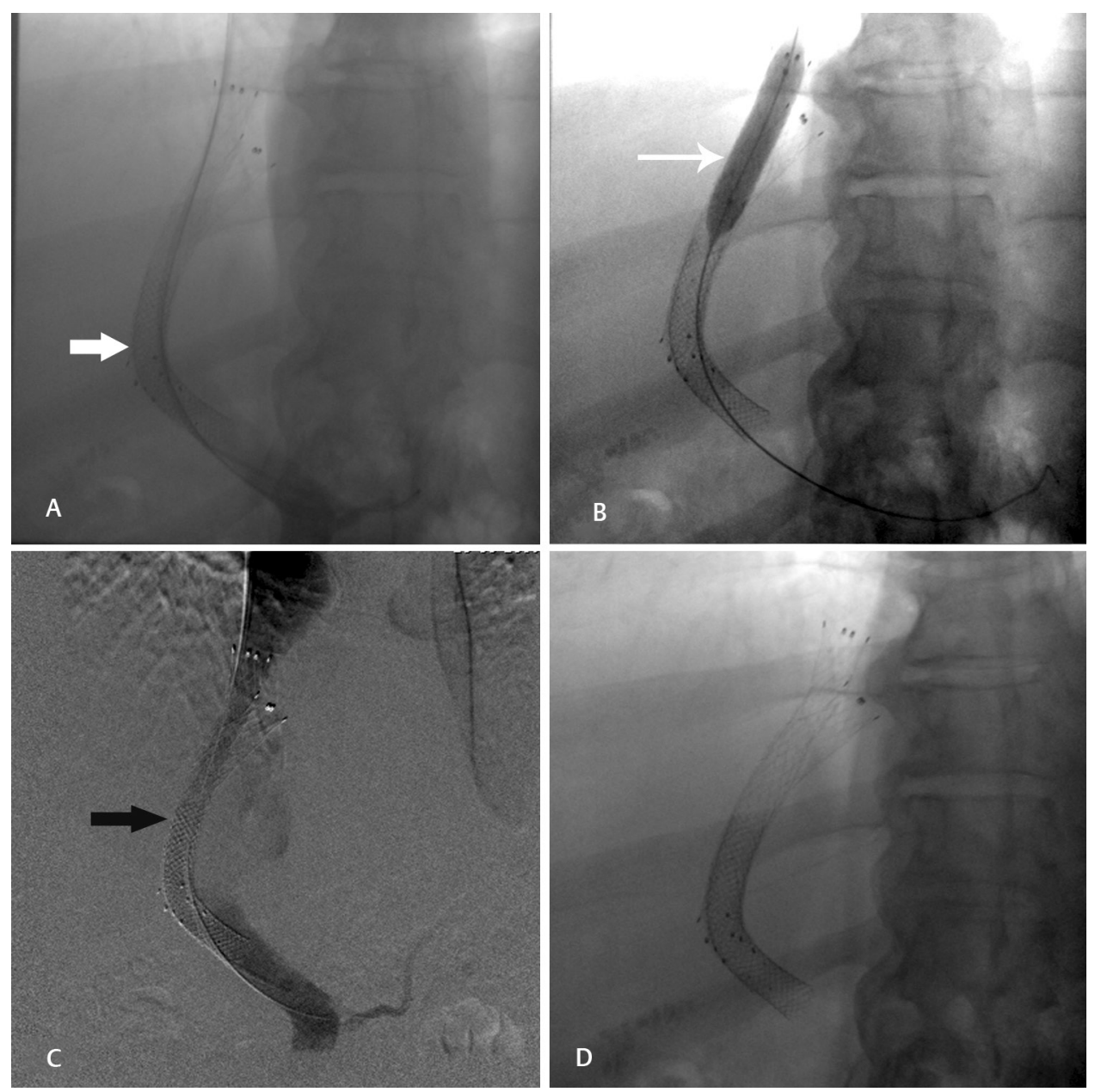

Fig. 1 (A) Digital subtraction angiography image shows access through the left internal jugular vein (IJV) with sheath seen on the left side (white arrow). (B) Fluoroscopy spot film shows that the RUPS set was then used to get access into stent directly through liver parenchyma through the lateral aspect of the direct intrahepatic portacaval shunt (DIPS) stent (white arrow). Direct entry and cannulation of the DIPS stent through the proximal inferior vena cava end (black arrow) was not possible due to chronic long-standing thrombosis and possible inadequate angulation due to left IJV approach. (C) Fluoroscopy spot films show the guidewire advanced along the tract through the lateral aspect of the stent (white arrow) till the splenic vein (black arrow). (D) The venogram confirms the portal vein position (white arrow) with absent flow through the blocked DIPS stent.

cannulation since we were approaching from the left side that may have altered the angulation of the DIPS with the IVC.

Using the needle of the RUPS set, a puncture using minimal hepatic tissue was made directly from the IVC to access

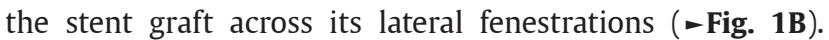
Hydrophilic guidewire and multipurpose catheter were advanced through the access and advanced into the portal vein. Direct portal pressure measured $34 \mathrm{~mm} \mathrm{Hg}$. A 5FR catheter was positioned in the distal splenic vein over an exchange length stiff guidewire (Amplatz Boston Scientific). Check

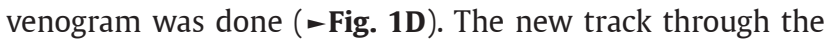
lateral aspect of the original DIPS stent was then dilated with a $10 \times 40 \mathrm{~mm}$ balloon ( - Fig. 2). A measuring pigtail catheter was used to measure the length of the new stent to be placed. A $10 \times 100$ FLUENCY covered stent and $10 \times 68$ uncovered wall stent was positioned from hepatic vein-IVC junction to right branch portal vein through liver parenchyma and the original DIPS stent graft ( $\boldsymbol{- \text { Fig. }}$ 3A). Balloon dilatation of the new stent was done ( $\boldsymbol{- \text { Fig. }}$ 3B). Check venogram revealed blood flow through the new stent and final spot confirmed its adequate position ( - Figs. $\mathbf{3 C}$ and $\mathbf{D}$ ). Final pressures were $22 \mathrm{~mm} \mathrm{Hg}$ in the main portal vein, $19 \mathrm{~mm} \mathrm{Hg}$ within the mid stent, $13 \mathrm{~mm} \mathrm{Hg}$ within the IVC, and $11 \mathrm{~mm} \mathrm{Hg}$ within the right atrium.

The following day the patient was started on injection enoxaparin and then discharged on anticoagulants. The new stent has shown adequate flow at 3- and 6-week follow-up Doppler examinations. The patient has no new complaints or signs of hepatic encephalopathy till present 6-month follow-up.

\section{Discussion}

TIPS is an effective, safe, and minimally invasive method for the treatment of complications related to portal hypertension. ${ }^{1}$ Petersen and Clark described DIPS in which a direct puncture through the caudate lobe is taken between the IVC and portal vein. ${ }^{2}$

Use of bare metallic stents in TIPS/DIPS has been associated with increased rates of TIPS dysfunction due to various 


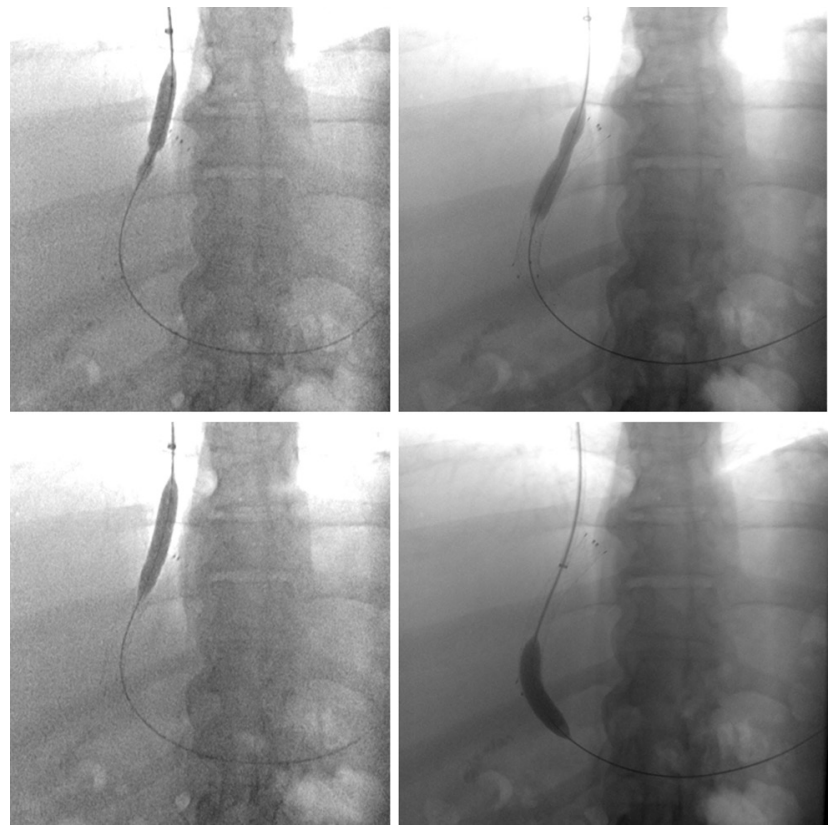

Fig. 2 (A-D) Fluoroscopy spot films show that the new track through the lateral aspect of the original direct intrahepatic portacaval shunt stent serially dilated with a $10 \times 40 \mathrm{~mm}$ balloon. factors. ${ }^{3}$ The creation of a shunt tract may transect bile radicals to create a bile-shunt communication. Suboptimal placement of the shunt at the hepatocaval junction also predisposes this site to turbulent blood flow with resultant shunt block. ${ }^{3}$ With the widespread use of polytetrafluoroethylene (PTFE)-covered stent grafts, the rate of shunt dysfunction has drastically reduced. Current covered stent graft has primary patency rates approximating 80 to $90 \%$ at 1 -year in contrast to patency rates below $50 \%$ for bare-metal stents. ${ }^{4}$ Even with the use of covered stent grafts, shunt blockage is a known complication that can be diagnosed on follow-up Doppler. $^{5}$

Once shunt dysfunction has been confirmed, multiple approaches have been described for revision. The most commonly applied technique for treating TIPS stenosis and occlusion is recanalization via the jugular vein approach with a hydrophilic guidewire. ${ }^{6}$ Alternative therapeutic strategies include transjugular access via a Colapinto needle for extra support and direct stent puncture via a RöschUchida needle. ${ }^{6}$ This is followed by balloon angioplasty, mechanical thrombectomy, catheter mediated thrombolysis, or placement of a new stent graft. A combined
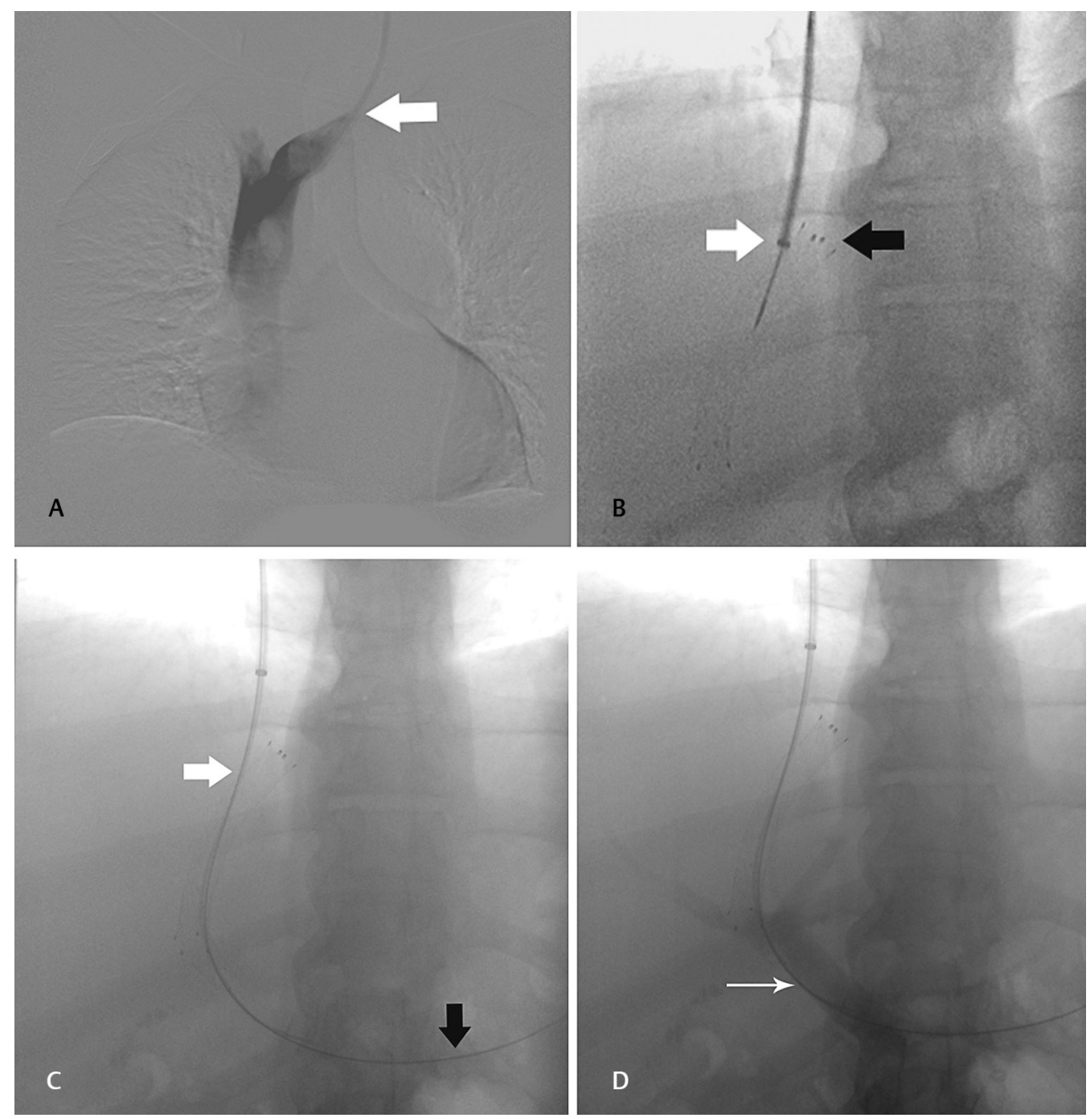

Fig. 3 (A) Fluoroscopy spot image shows new stent deployed through lateral aspect of the original direct intrahepatic portacaval shunt stent (white arrow). (B) Balloon dilatation (white arrow) of the new deployed stent tract was done. (C) Venogram reveals adequate passage of contrast through the new stent (black arrow). (D) Final spot film reveals satisfactory position of the new stent. 
pull-through wire technique with combined transhepatic and transjugular approach has also been described. ${ }^{7}$

In 1992, Haskal et al first reported creation of a second parallel TIPS tract to reduce the portosystemic pressure gradient. ${ }^{8}$ More recently, He et $\mathrm{al}^{9}$ and Raissi et $\mathrm{al}^{10}$ have also described the feasibility and effectiveness of parallel TIPS. Parallel TIPS involves the creation of a second new tract, usually from the right hepatic vein to the right branch of the portal vein, parallel to the first stent and using the first stent as a marker. The second stent can also be placed from the middle hepatic vein to the left portal vein. ${ }^{11}$ However, if the hepatic veins are not accessible, then a new shunt can be created directly from the IVC to the portal vein. ${ }^{9}$ If available, Viatorr stent graft can be used for performing the parallel TIPS/DIPS procedure. If not, then a combination of PTFE covered and uncovered stent can be used. ${ }^{10}$

We describe a unique technique where in cases of TIPS/ DIPS revision a new tract can be created through a lateral direct puncture across the fenestrations of the existing dysfunctioning stent graft. This technique can be used when it is not possible to gain access into the proximal hepatic venous end of the blocked stent. Instead of using a long completely new tract through the hepatic parenchyma that has been described in the parallel TIPS technique in literature, we propose using only a sliver of hepatic tissue through direct puncture from the IVC and entry across the lateral fenestrations of the dysfunctioning shunt. Theoretically, this technique can offer advantages over a parallel TIPS technique since reduced hepatic parenchyma in the shunt tract will reduce complications. Technical problems encountered during this procedure may include difficult entry into the shunt through the stent mesh and difficulty in tract dilatation at this entry point. Despite technical challenges, this technique can serve as an alternate to conventional parallel TIPS/DIPS. Further studies and follow-up are required to validate its long-term patency and clinical outcomes.

\section{Funding}

This study was not supported by any funding.

\section{Ethical Approval}

For this type of study, formal consent is not required.

\section{Informed Consent}

For this type of study, informed consent is not required.

\section{Conflict of Interest}

The authors declare that they have no conflict of interest.

\section{Acknowledgments}

None.

\section{References}

1 Parvinian A, Bui JT, Knuttinen MG, Minocha J, Gaba RC. Transjugular intrahepatic portosystemic shunt for the treatment of medically refractory ascites. Diagn Interv Radiol 2014;20(1):58-64

2 Petersen BD, Clark TW. Direct intrahepatic portocaval shunt. Tech Vasc Interv Radiol 2008;11(4):230-234

3 Cura M, Cura A, Suri R, El-Merhi F, Lopera J, Kroma G. Causes of TIPS dysfunction. AJR Am J Roentgenol 2008;191(6):1751-1757

4 Weber CN, Nadolski GJ, White SB, et al. Long-term patency and clinical analysis of expanded polytetrafluoroethylene-covered transjugular intrahepatic portosystemic shunt stent grafts. J Vasc Interv Radiol 2015;26(9):1257-1265, quiz 1265

5 Darcy M. Evaluation and management of transjugular intrahepatic portosystemic shunts. AJR Am J Roentgenol 2012;199(4):730-736 Review. Erratum in: AJR Am J Roentgenol. 2013 Jan;200(1):232. PubMed PMID: 22997362

6 Spiliopoulos S, Vasiniotis Kamarinos N, Konstantos C, et al. Recanalization of occluded transjugular intrahepatic portosystemic shunts using the Rösch-Uchida stiffening cannula. Cardiovasc Intervent Radiol 2018;41(5):799-803

7 Tanaka T, Günther RW, Isfort P, Kichikawa K, Mahnken AH. Pull-through technique for recanalization of occluded portosystemic shunts (TIPS): technical note and review of the literature. Cardiovasc Intervent Radiol 2011;34(2):406-412

8 Haskal ZJ, Ring EJ, LaBerge JM, et al. Role of parallel transjugular intrahepatic portosystemic shunts in patients with persistent portal hypertension. Radiology 1992;185(3):813-817

9 He FL, Wang L, Yue ZD, Zhao HW, Liu FQ. Parallel transjugular intrahepatic portosystemic shunt for controlling portal hypertension complications in cirrhotic patients. World J Gastroenterol 2014;20(33):11835-11839

10 Raissi D, Yu Q, Nisiewicz M, Krohmer S. Parallel transjugular intrahepatic portosystemic shunt with Viatorr ${ }^{\circledR}$ stents for primary TIPS insufficiency: Case series and review of literature. World J Hepatol 2019;11(2):217-225

11 Rathod K, Popat B, Barai P, Amrapurkar D. Parallel transjugular intrahepatic portosystemic shunt (TIPS) creation in a patient with a preexisting unsalvageable occluded tips and refractory variceal bleeding. J Clin Intervent Radiol ISVIR 2017;1:40-42 